\title{
Ionization of Fast Rydberg Atoms in Longitudinal and Transverse Electric Fields
}

\author{
S. A. Aseyev, Yu. A. Kudryavtsev, V. V. Petrunin \\ Institute of Spectroscopy, Russian Academy of Sciences, 142092 Troitzk, Moscow Region, Russia (Fax: + 7-095/334-0886)
}

Received in revised form 14 December 1992/Accepted 16 December 1992

\begin{abstract}
The main properties of longitudinal and transverse electric field ionizers for fast Rydberg atoms $n=$ $21-40$ have been investigated. The dispersion and the background due to collisional processes between fast atoms and residual gas molecules have been measured and calculated. The kinetic energy spread of ions formed by field ionization of Rydberg atoms and their trajectories have been calculated. The potassium beam energy was $3.9 \mathrm{keV}$.
\end{abstract}

PACS: $32.80 . \mathrm{Rm}$, 32.80.Fb

The method of collinear resonant laser photoionization of atoms in an accelerated beam can be applied to detect ultralow concentration densities (less than $10^{-9}$ ) of rare isotopes amidst the abundant main isotopes of the same element $[1,2]$. The idea of this method consists in the isotope-selective stepwise excitation of the fast isotopic atoms of interest to a Rydberg state by means of laser radiation propagating collinear to the atomic beam. The atomic energy being of the order of $10 \mathrm{keV}$, the kinematic isotope shift is usually 10 to 100 times larger than the natural isotope shift in any atomic transition. The absorption linewidth in this case can be reduced to the natural linewidth $[3,4]$. Thus, this method is potentially capable of an extremely high selectivity in the laser excitation of rare isotopic atoms to a Rydberg state. The Rydberg atoms are ionized by the electric field of an ionizer and deflected onto the detector. The field ionization through a Rydberg state is more preferable because this makes it possible to reduce considerably the background due to the collisional ionization of fast atoms on the residual gas molecules. The field ionizer, in this case, should be constructed so that the detector can receive ions only from a small region of the electric field where Rydberg atoms are ionized. The ions formed beyond this region should not reach the detector. It has been shown $[2,5]$ that the excitation of fast helium atoms to Rydberg states resulting from their collisions with the residual gas molecules makes a considerable contribution to the background, too. Thus, the important characteristic property of the ionizer is the ability to separate the signal ions from the collisional background. This paper presents the results of our studies of two ionizers - with longitudinal and transverse directions of electric field relative to the atomic beam trajectory.

The longitudinal and transverse ionization schemes have been used before to investigate the distribution of the Rydberg atoms formed in the process of charge exchange $[6,7]$ with respect to the principal quantum number. The ionization in the inhomogeneous-field stripper [8] was used to study the distribution of the atomic states, resulting from the charge exchange between the ions and highly-excited $\mathrm{Na}$ atoms [9]. The longitudinal $[10,11]$ and transverse [12-14] ionization schemes were applied to study Rydberg atoms excited by laser radiation as well as to measure the cross-sections of collisional excitation to Rydberg states of fast $\mathrm{He}[15]$ and $\mathrm{H}$ [16] atoms.

\section{Experimental Setup}

The experimental setup was described in detail in $[5,12]$. The continuous beam of $\mathrm{K}$ atoms was obtained by resonant charge exchange between the fast $\mathrm{K}$ ions and atoms in potassium vapor. The atomic beam energy was 3.9 $\mathrm{keV}$. The fast $\mathrm{K}$ atoms in the beam were excited to Rydberg states in two steps according to the scheme $4 S_{1 / 2} \rightarrow 4 P_{3 / 2} \rightarrow n D_{5 / 2}$ (or $n S_{1 / 2}$ ) by means of laser radiation counterpropagating to the beam in a field-free region, $1 \mathrm{~m}$ long. The first-step excitation laser had $\lambda_{1}=765 \mathrm{~nm}$. The second-step excitation laser wavelength was tuned over the range $456-461 \mathrm{~nm}$ and so the $\mathrm{K}$ atoms could be excited to the Rydberg states with $n=20-40$. The dye lasers were pumped by an $\mathrm{XeCl}$ excimer laser with a $20 \mathrm{~Hz}$ pulse repetition rate. The bandwidths of laser radiation in the first and second excitation steps were $0.5 \mathrm{~cm}^{-1}$ and $0.8 \mathrm{~cm}^{-1}$, respectively.

The Rydberg atoms were ionized in the ionizer, and the resulting ions were deflected onto the detector, i.e. a secondary electron multiplier, placed at angle $\alpha=22^{\circ}$ relative to the atomic beam axis. A gated counting system was 


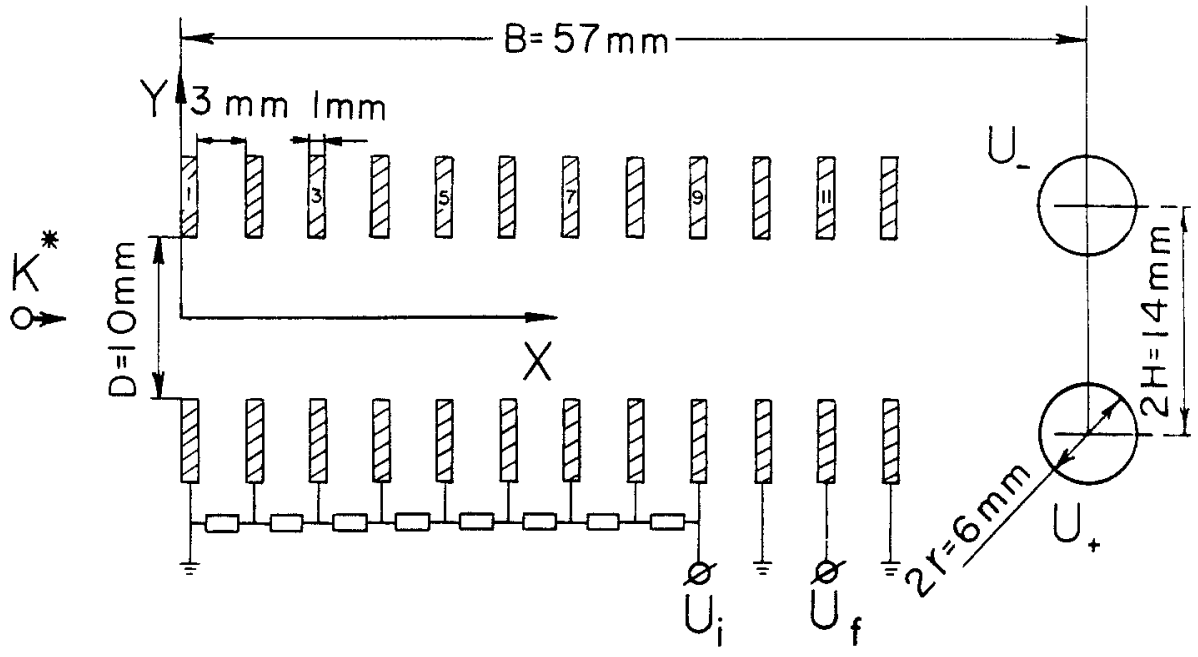

Fig. 1. Scheme of the ionizer used to measure the background signal resulting from the collisional processes; the signal ions from the entire fieldfree region was measured using a recording system which integrated the current passing through the multiplier after each laser pulse.

\section{Ionizer}

The ionizer consisted of 12 equally-spaced coaxial diaphragms with their planes perpendicular to the atomic beam axis. Its dimensions are presented in Fig. 1. The thickness of the diaphragms $\delta=1 \mathrm{~mm}$, the distance between them $\Delta=3 \mathrm{~mm}$. The excited potassium atoms entered the ionizer on the side of the first diaphragm. Under transverse ionization the potentials of all the coaxial diaphragms are equal to zero. The Rydberg atoms are ionized in the electric field formed by two cylinders, and the resulting ions are deflected by the same field onto the detector. In this case there is only a transverse component of the electric field on the ionizer axis. Under longitudinal ionization the potential $U_{\mathrm{i}}$ is fed to the 9th diaphragm. The potentials of the first eight diaphragms are $U_{k}=U_{\mathrm{i}}(k-1) / 8$, where $k=1-8$. The Rydberg atoms formed by laser radiation are ionized between the 9 th and 10th diaphragms where the electric field strength is maximal. There is only a longitudinal component of the electric field on the ionizer axis. The ions formed in the ionizer can be focused by a single lens, consisting of diaphragms 10 12. The cylindrical electrodes at potentials of $U_{+}$and $U_{-}$ act as a deflector directing the ions onto the detector.

\section{Ionizer Electric Field}

The distribution of the potential formed by a system of coaxial diaphragms was calculated by solving the Laplace equation written in a cylindrical coordinate system $r, x, \theta$ for a field with rotational symmetry [17]:

$\nabla^{2} U=\frac{1}{r} \frac{\partial}{\partial r}\left(r \frac{\partial U}{\partial r}\right)+\frac{\partial^{2} U}{\partial x^{2}}=0$.

The distribution of the potential $U_{\text {int }}$ in the gap between diaphragms, with $r=D / 2$, was assumed to be linear. It can be written analytically in the coordinates $x, y$ as a Fourier series

$U(x, y=D / 2)=\sum_{n} \varphi_{n} \sin (\pi n x / L)$,

where

$\varphi_{n}=\frac{2}{L} \int_{0}^{L} U_{\mathrm{int}} \cdot \sin (\pi n x / L) d x, \quad L=11(\Delta+\delta)+2 \delta$,

$L$ is the dimension of the area where the ionizer electric field is accumulated. The distribution of the potential inside the system of coaxial diaphragms has the form

$U(x, y)=\sum_{n} A_{n} I_{0}(\pi n y / L) \sin (\pi n x / L)$,

where $A_{n}=\varphi_{n} / I_{0}\left(\frac{\pi n D}{2 L}\right), I_{0}$ is the zeroth-order modified Bessel function. The electric field strength components in this case will be

$E_{x}(x, y)=-\frac{\partial U}{\partial x}=-\sum_{n} A_{n} \cdot I_{0}\left(\frac{\pi n y}{L}\right) \cdot \frac{\pi n}{L} \cdot \cos \left(\frac{\pi n x}{L}\right)$,
$E_{y}(x, y)=-\frac{\partial U}{\partial y}=-\sum_{n} A_{n} \cdot I_{1}\left(\frac{\pi n y}{L}\right) \cdot \frac{\pi n}{L} \cdot \sin \left(\frac{\pi n x}{L}\right)$,

where $I_{1}$ is the first-order modified Bessel function. The potential and the electric field strength distribution were calculated with a computer. The electric field calculation accuracy depends on the number of terms of the Fourier series. As the number of terms varies from 20 to 50 , the results of calculation do not change considerably, and therefore the Fourier series in our calculation was restricted to 50 terms. Figure 2 shows the calculated distributions of the electric field potential and the absolute field strength on the ionizer axis with $U_{\mathrm{i}}=2000 \mathrm{~V}$ and $U_{\mathrm{f}}=-2000 \mathrm{~V}$. The focusing electrode at potential of $U_{\mathrm{f}}$ makes it possible to focus the resulting ions onto the detector. It can be seen that the field strength is constant between diaphragms 2 and 6 and maximal near diaphragm 10 .

The electric field and the distribution of the potential formed by two cylindrical electrodes at potentials of $U_{+}$ and $U_{-}$, when $\left|U_{-}\right|=U_{+}$, can be written in the form [18] 


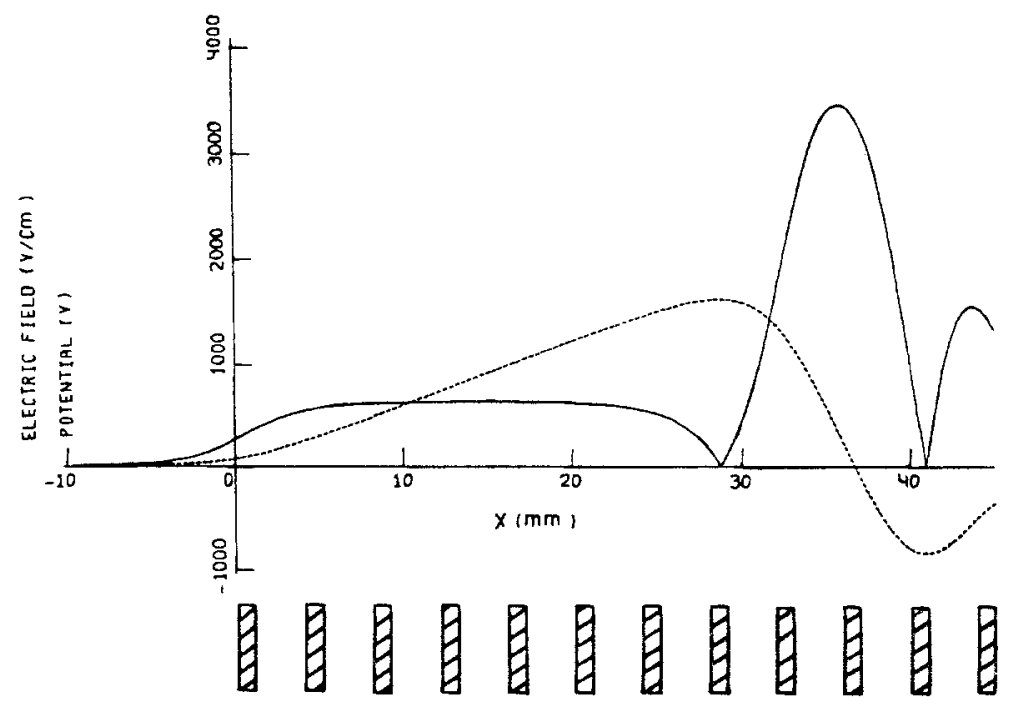

Fig. 2. Calculated dependences of absolute field strength (solid line) and potential (dashed line) of electric field on the ionizer axis on the $x$-coordinate for $U_{\mathrm{i}}=2000 \mathrm{~V}$, $U_{\mathrm{f}}=-2000 \mathrm{~V}$
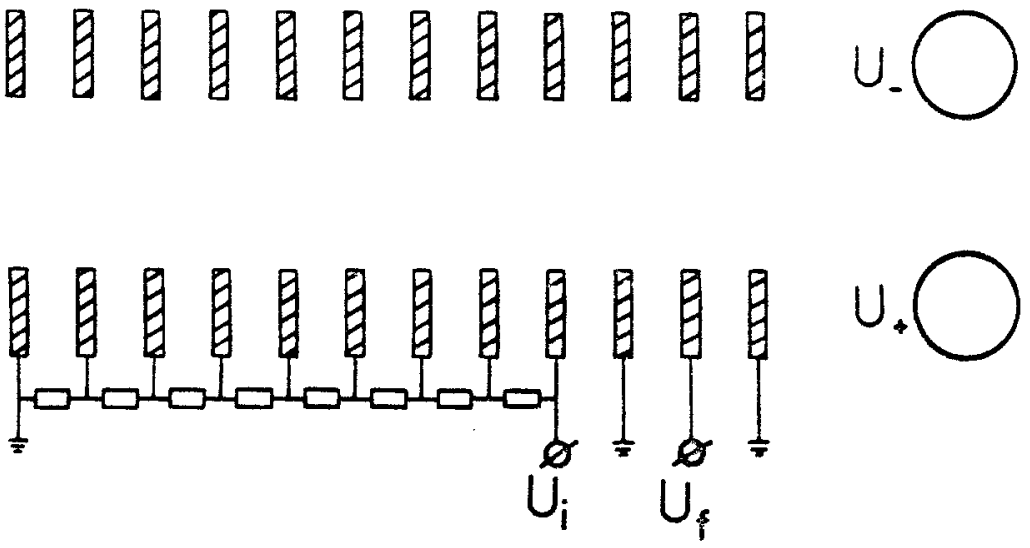

$E_{x}(x, y)=K x\left(1 / a_{1}^{2}-1 / a_{2}^{2}\right)$

$E_{y}(x, y)=K\left[(y+h) / a_{1}^{2}+(h-y) / a_{2}^{2}\right]$,

$U(x, y)=K \ln \left(a_{2} / a_{1}\right)$,

where $h=\sqrt{H^{2}-r^{2}}, K=U_{+} / \ln [(H+h) / r]$,

$a_{1}=\sqrt{(x-B)^{2}+(y+h)^{2}}$,

$a_{2}=\sqrt{(x-B)^{2}+(y-h)^{2}}$.

The solid line in Fig. 3 shows the calculated dependence of the field strength on the ionizer axis. The maximum field strength corresponds to $x=B=57 \mathrm{~mm}$. The potential on the ionizer axis is equal to zero.

The field strength in which the Rydberg atoms become ionized depends significantly on the evolution of the atoms in the growing electric field $[19,20]$. The Stark sublevels of a real atom cannot cross. A region of level quasicrossing is formed. The width of the resulting enerqy gap depends on the extent to which the levels are coupled. An atom will enter the level quasicrossing region adiabatically when the field increases sufficiently slowly, and the energy gap is large. In this case the point where Rydberg atoms with the effective principal quantum number $n^{*}$ are ionized is approximately determined by the critical electric field strength $E_{\mathrm{cr}}=3.2 \times 10^{8} / n^{* 4} \mathrm{~V} / \mathrm{cm}[19,20]$. Unlike in our experiments with $\mathrm{He}$ [5], for $\mathrm{K}$ we have observed only adiabatical ionization of Rydberg atoms. The position of the signal corresponding to the excitation of the $n D$ state was the same as the position of the signal from $(n+2) S$ state.

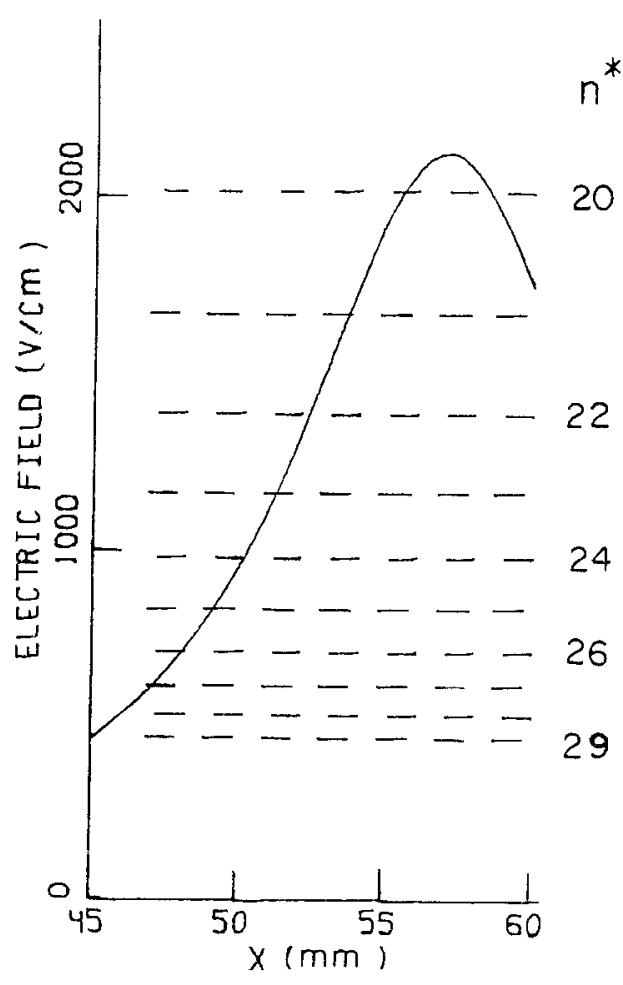

Fig. 3. Calculated dependences of absolute field strength of electric field on the ionizer axis on the $x$-coordinate for $U_{+}=$ $-U_{-}=1000 \mathrm{~V}$ (solid line) and critical field strength for different values of the effective principal quantum number $n^{*}$ (dashed lines) 


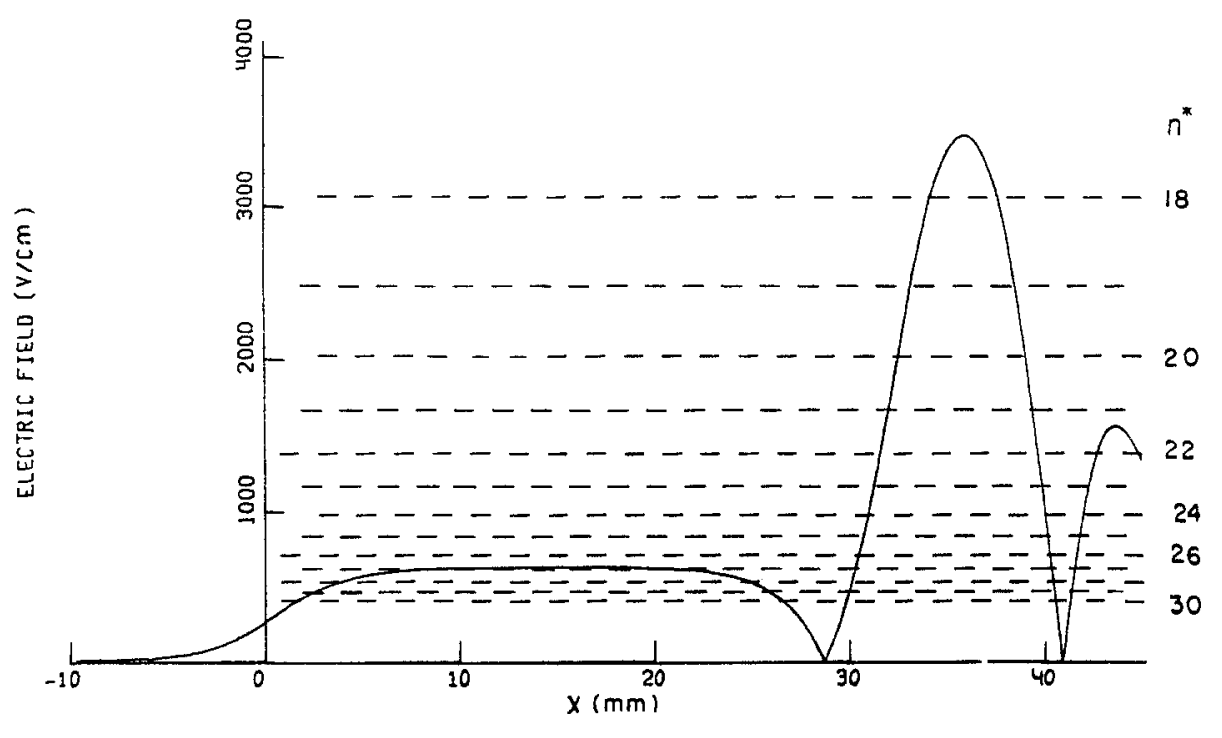

Fig. 4. Calculated dependence of absolute field strength of electric field on the ionizer axis on the $x$-coordinate for $U_{\mathrm{i}}=2000 \mathrm{~V}, U_{\mathrm{f}}=-2000 \mathrm{~V}$ (solid line) and critical field strength for different values of the effective principal quantum number $n^{*}$ (dashed lines)
The dashed lines in Fig. 3 correspond to the critical field strength for $n^{*}=20$ to 29 . Figure 4 shows similar dependences for a longitudinal-field ionizer. With the ionizing potential of $U_{\mathrm{i}}=2000 \mathrm{~V}$, atoms with $n^{*}$ more than $18 \mathrm{can}$ be ionized. It can be seen that, if $U_{\mathrm{i}}=2000 \mathrm{~V}$, atoms with $n^{*}$ more than 27 are ionized at the entry of the ionizer between diaphragms 1 and 2, and atoms with $n^{*}$ less than 27 are ionized in a region with a higher field strength between diaphragms 9 and 10 .

\section{Ion Trajectory Calculation}

Ion trajectories are calculated by solving the system of differential equations using the Runge--Kutta method by means of a computer.

$$
\left\{\begin{array}{l}
\dot{x}=v_{x}, \\
\dot{y}=v_{y}, \\
\dot{v}_{x}=\frac{e}{M} E_{x}, \\
\dot{v}_{y}=\frac{e}{M} E_{y},
\end{array}\right.
$$

where $U_{x}, U_{y}$ are the projections of ion velocity on the axes $x$ and $y, e$ is the ion charge, and $M$ is the ion mass. The calculations were carried out for atoms on the ionizer axis and at distances of $+1 \mathrm{~mm}$ and $-1 \mathrm{~mm}$ from the axis. Figure 5 shows ion trajectories in a longitudinal ionizer. Figure $5 \mathrm{a}$ presents the trajectories of the ions formed by field ionization of atoms with $n^{*}=25$. Figure $5 \mathrm{~b}$ presents the trajectories of the ions flying into the ionizer with the same values of $U_{\mathrm{i}}, U_{\mathrm{f}}, U_{+}, U_{-}$. These ions formed in the field-free region due to collisional ionization of neutral $\mathrm{K}$ atoms by the residual gas molecules. It can be seen that the deflection angle in Fig. $5 \mathrm{~b}$ is larger, because the Rydberg atoms are subjected to the action of the electric field only after ionization. The final kinetic energy of the resulting ions in this case is higher than the initial energy of the atoms by about $1.5 \mathrm{keV}$ (Fig. 2). The kinetic energy of the ions, formed in a field-free region, does not change.
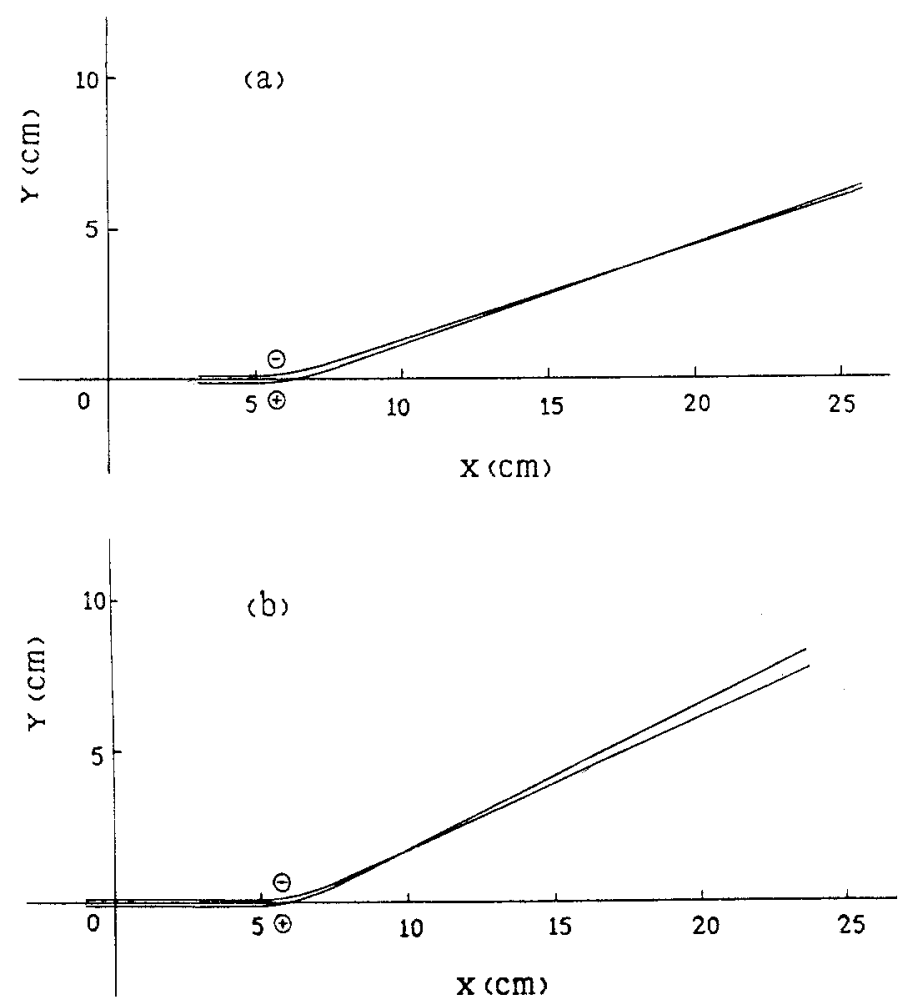

Fig. 5. Calculated trajectories of ions in a longitudinal-field ionizer for $U_{\mathrm{i}}=2000 \mathrm{~V}, U_{\mathrm{f}}=-2000 \mathrm{~V}, U_{+}=-U_{-}=1000 \mathrm{~V}$ : (a) formed by ionization from Rydberg atoms with $n^{*}=25$; (b) formed in the field-free region

It can be also seen that after passing the ionizer the divergence of the initial ion beam is larger than that of the ions formed from atoms with $n^{*}=25$. Similar ion trajectories in a transverse-field ionizer are presented in Fig. 6. For the trajectories given in Fig. 6a the field begins acting at a point where the field strength is critical for atoms with $n^{*}=25$. In Fig. $6 \mathrm{~b}$ the ion is subjected to the action of the field starting from $x=40$. It can be seen, that the deflection angle for ions created in the field-free region is larger than for the ions formed from atoms with $n^{*}=25$. 

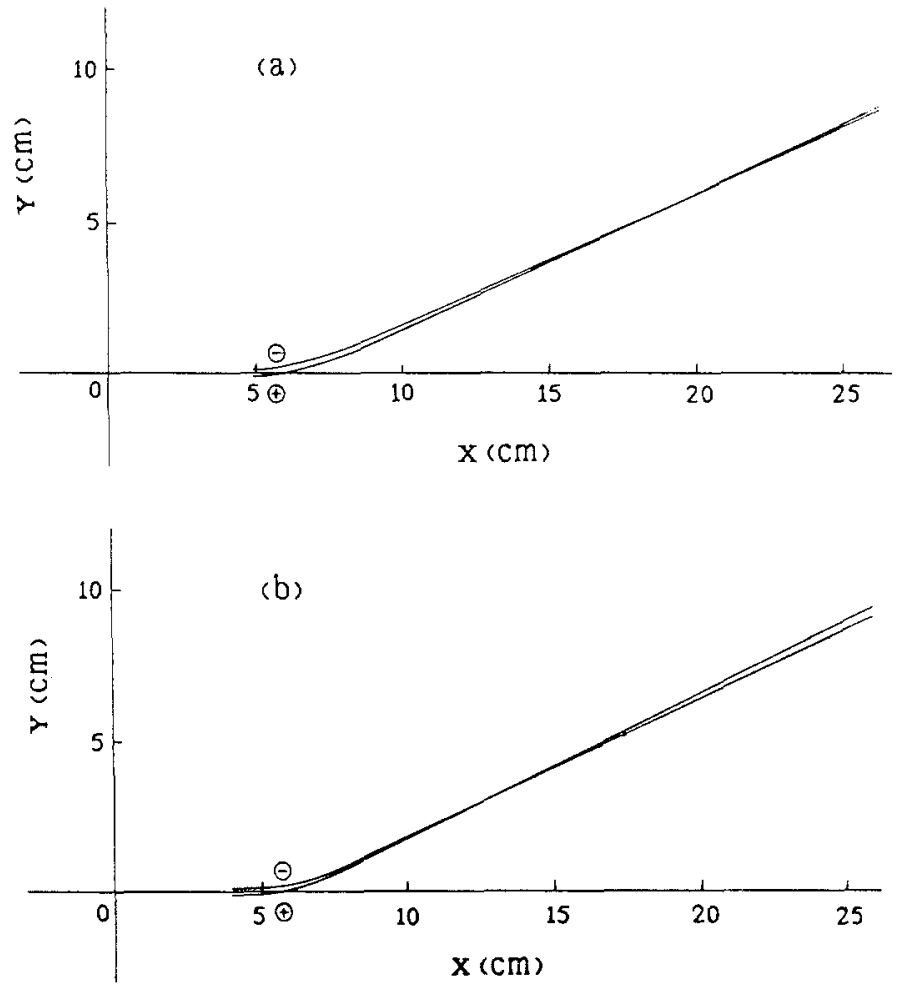

Fig. 6. Calculated trajectories of ions in a transverse-field ionizer for $U_{+}=-U_{-}=1000 \mathrm{~V}$ : (a) formed by ionization from Rydberg atoms with $n^{*}=25$; (b) formed in the field-free region

\section{Ionizer Dispersion}

In both ionizers atoms with different $n^{*}$ are ionized at different points of the ionizer. If the angle of ion deflection $\alpha$ is fixed, the potentials $U_{+}, U_{-}\left(U_{+}=-U_{-}\right)$, which should be applied to the cylindrical electrodes in order to deflect the ions onto the detector, are different for various $n^{*}$. The dependence $U_{-}\left(n^{*}\right)\left(U_{+}=-U_{-}\right)$for the longitudinal-field ionizer is determined by the potential distribution. The solid lines in Fig. 7 show the potentials at the cylindrical electrodes needed to deflect the ionized atoms at the angle $\alpha=22^{\circ}$ as a function of the effective principal quantum number $n *$ for ionizers with transverse (Fig. 7a) and longitudinal (Fig. $7 \mathrm{~b}$ ) fields. The calculation was done for the atoms on the ionizer axis. The experimental results are marked with asterisks. One can see that there is a good agreement between calculated and experimental data and, also, that the curves for different ionizers are qualitatively different. For the transverse-field ionizer (Fig. 7a) the potential $\left|U_{-}\right|$decreases smoothly with an increase in $n^{*}$. This is due to the fact that atoms with large $n^{*}$ are ionized by an electric field with a lower strength, i.e. farther from the cylindrical electrodes, and longer subjected to the deflecting action of the cylinder field. In the longitudinal-field ionizer the situation is more complicated (Fig. 7b). For atoms with $n^{*} \geq 27$ the field strength is higher than critical strength even at the entry into the ionizer (diaphragms 1, 2). Atoms with $n^{*} \leq 28$ can be ionized only between diaphragms 9 and 10 . As $n^{*}$ drops from 28 to 21 , the required potential $\left|U_{-}\right|$decreases. This is due to the fact that ionization occurs at points with lower potentials (Fig. 2). Hence,
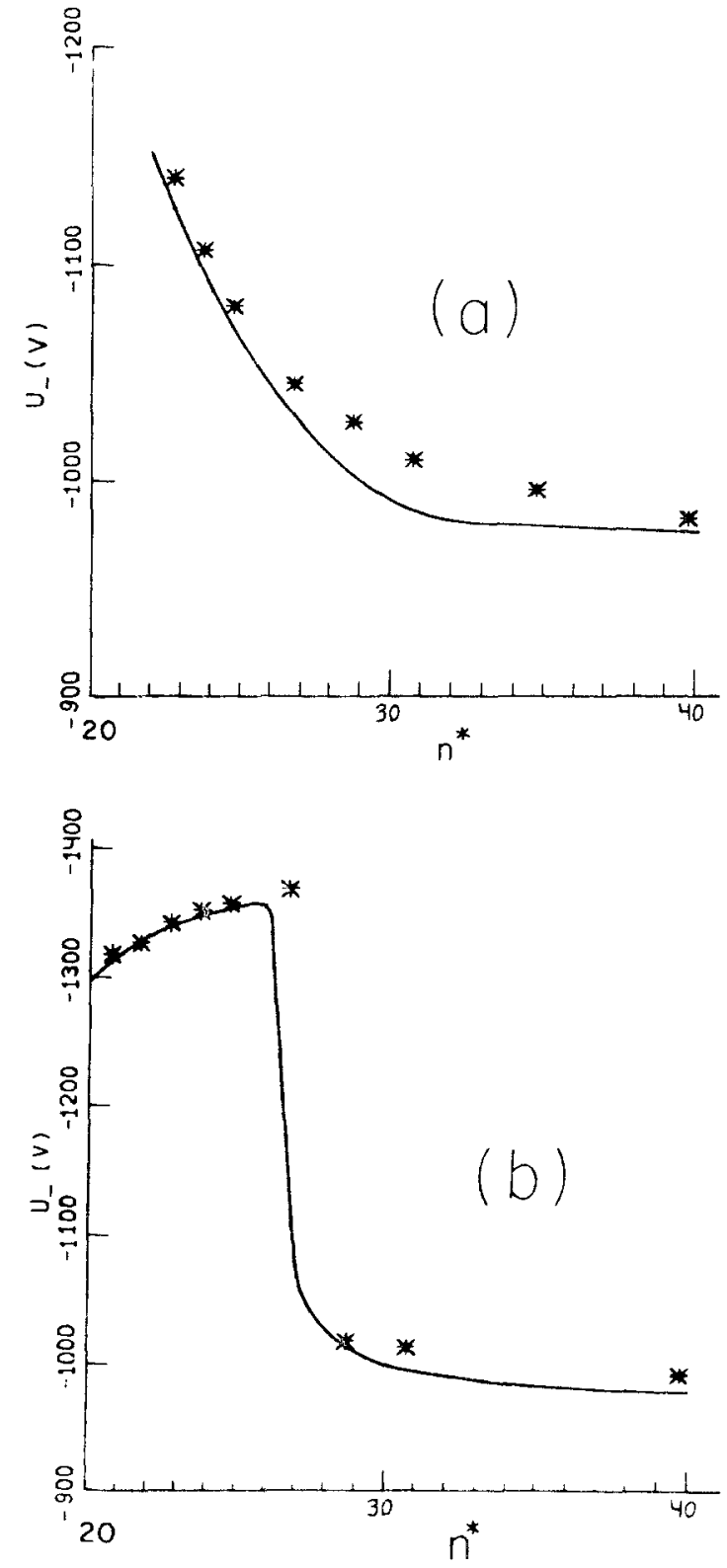

Fig. 7. Dependences of the potentials $U_{-}=-U_{+}$at the cylindrical electrodes, needed for deflecting the ionized atoms at the angle of $\alpha=22^{\circ}$ on the effective principal quantum number $n^{*}$ for transverse (a) and longitudinal (b) ionization if $U_{\mathrm{i}}=2000 \mathrm{~V}$, $U_{\mathrm{f}}=-2000 \mathrm{~V}$. Solid curve: calculation, asterisks experimental data

the resultant ions have a lower energy and they need a lower potential at the cylinders $\left|U_{-}\right|$to be deflected onto the detector. It can be seen from Fig. 7 that, with $n^{*} \rightarrow+\infty$, $U_{-}$is the same for the both ionizers.

\section{Energy Spread of Resultant Ions}

For detecting ultra rare isotopes one can use further separation of ions resulting from field ionization of Rydberg atoms. The kinetic energy spread of ions in both ionizers depending on $n^{*}$ is a very important quantity for joining the ionizer with the mass separator. Table 1 presents the 
Table 1. Calculated values of potentials [V] of the electric field at points, where the field strength is critical for different $n^{*}$, in the case of longitudinal (a) and transverse (b) ionization on the ionizer axis and at a distance of $1 \mathrm{~mm}$ from the axis, as well as the relative energy spread of resultant ions $\Delta \varepsilon[\%]$ with an initial atomic beam energy of $3.9 \mathrm{keV}$

\begin{tabular}{|c|c|c|c|c|}
\hline a) & $n^{*}$ & $y=0 \mathrm{~mm}$ & $y=1 \mathrm{~mm}$ & $\Delta \varepsilon[\%]$ \\
\hline & 20 & 1310 & 1338 & 0.5 \\
\hline & 30 & 116 & 111 & 0.1 \\
\hline \multirow[t]{3}{*}{ b) } & $n^{*}$ & $y=-1 \mathrm{~mm}$ & $y=1 \mathrm{~mm}$ & $\Delta \varepsilon[\%]$ \\
\hline & 20 & 200 & -200 & 10.3 \\
\hline & 30 & 40 & -40 & 2.1 \\
\hline
\end{tabular}

results of calculation for the potentials of the points where the field strength is critical for different values of $n^{*}$ and the relative energy spread for ions if the initial beam energy is $3.9 \mathrm{keV}$. It was assumed that ionization occurs very fast, and the ion energy spread due to the final ionization rate of atoms is small.

\section{Collisional Ionization of Atoms}

Collisional excitation and ionization of the fast atoms of the abundant isotope by the residual gas molecules form a serious limitation for the method of detecting the rare isotopes by means of collinear laser photoionization of atoms in an accelerated beam. The cross sections of collisional excitation to Rydberg states are very small, $\sim 10^{-20}$ $\mathrm{cm}^{2}[15,16]$. These atoms are formed along the whole field-free region of excitatlon and cannot be distinguished from the rare isotopic atoms in the Rydberg state since they are ionized at the same points of the ionizer as the rare isotopic atoms are. The second cause of background is collisional ionization of fast atoms at the points where field ionization of the rare isotopic atoms takes place. The cross section of collisional ionization is approximately $10^{3}$ times larger than that of collisional excitation to a Rydberg state. The value of this background depends on the design of the ionizer. This collisional background is proportional to the length of the region from which the ions can reach the detector.

The ions formed by collisions in the field-free region can be extracted from the beam by a filter deflector at the entry into the ionizer.

The background resulting from collisional ionization was calculated in the following manner. For every point of the ionizer we calculated the potential $U_{-}\left(U_{+}=-U_{-}\right)$ that must be applied to the cylinders in order to deflect the ions, formed at a given point, to the detector. Such a dependence for longitudinal ionization is given in Fig. 8 . The minimal potential $\left|U_{-}\right|$corresponds to the point for which the final kinetic energy of the resulting ions is minimal (Fig. 2) and the peak of the potential $\left|U_{-}\right|$corresponds to the point where the potential in Fig. 2 is maximal $(x \simeq 29 \mathrm{~mm})$. For transverse ionization this dependence is a monotonically increasing curve. It is assumed that the value of background for a given potential $U_{-}$is proportional to the length of the region for which the ions formed

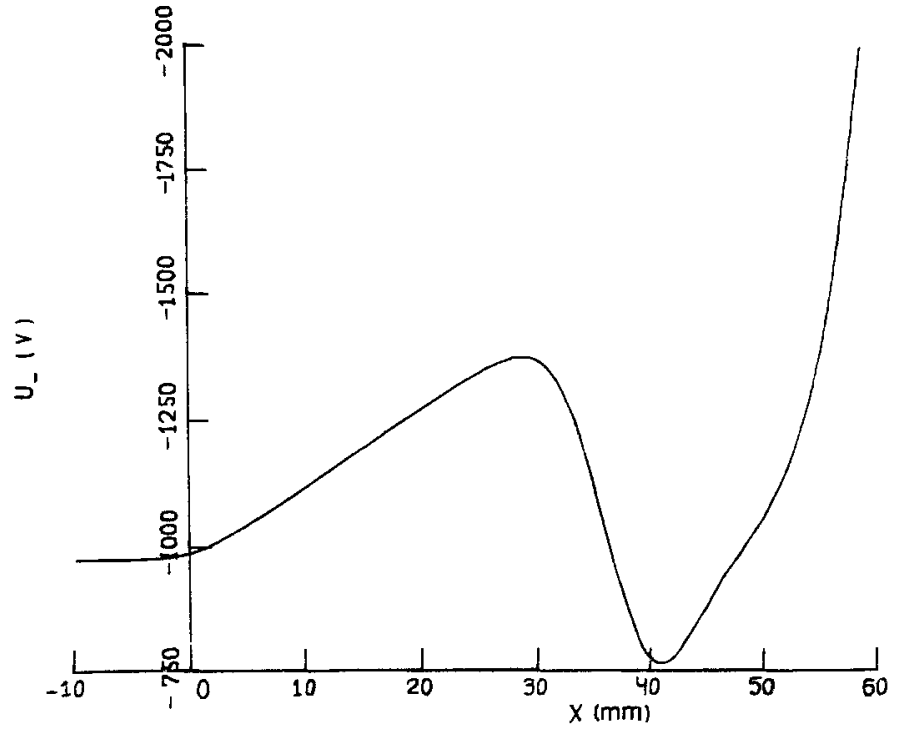

Fig. 8. Calculated dependences of the potentials $U_{-}=-U_{+}$at the cylindrical electrodes, needed for deflecting the ions formed at the point with the $x$-coordinate at the angle of $\alpha=22^{\circ}$ on the $x$-coordinate. $U_{\mathrm{i}}=2000 \mathrm{~V}, U_{\mathrm{f}}=-2000 \mathrm{~V}$

by collisions have the same final energy. The calculated dependence of the background due to collisional ionization in the region of the ionizer on the voltage at the cylinders $U_{-}$is given in Fig. 9a for longitudinal ionization. It was assumed that ions with an energy spread of $10 \mathrm{eV}$ can reach the detector. The highest peak $\left(U_{-}=\right.$ $-970 \mathrm{~V}$ ) corresponds to the ions from the region with $x<0$ and from small parts with $x=36 \mathrm{~mm}$ and $47 \mathrm{~mm}$. The main contribution to the peak at $U_{-}=-1360 \mathrm{~V}$ comes from the ions formed in the region corresponding to the maximum in Fig. $8(x=29 \mathrm{~mm})$. Figure $9 \mathrm{~b}$ shows the experimental dependence of the background resulting from collisional processes on voltage $U_{-}$. One can see that the positions of the peaks in Fig. $9 \mathrm{~b}$ are in satisfactory agreement with the calculated values. The higher value of the experimental background at $U_{-}=-970 \mathrm{~V}$ is due to the ions from the field-free region. The calculated and experimental dependences of the background for the transverse-field ionizer are given in Fig. 10a and Fig. 10b, respectively. It can be also seen that the position of the peak and its shape are in satisfactory agreement with calculated results.

\section{Conclusions}

The properties of longitudinal and transverse ionizers for fast Rydberg atoms have been studied experimentally and theoretically. Satisfactory agreement between calculated results and experimental data has been obtained for such properties as dispersion and behavior of the background due to collisional processes between fast atoms and residual gas molecules. The energy spread of the ions formed by field ionization of Rydberg atoms and their trajectories have been calculated. The energy spread of the ions formed after ionization of Rydberg atoms in the longitudinal scheme is smaller than in the transverse one. Lon- 

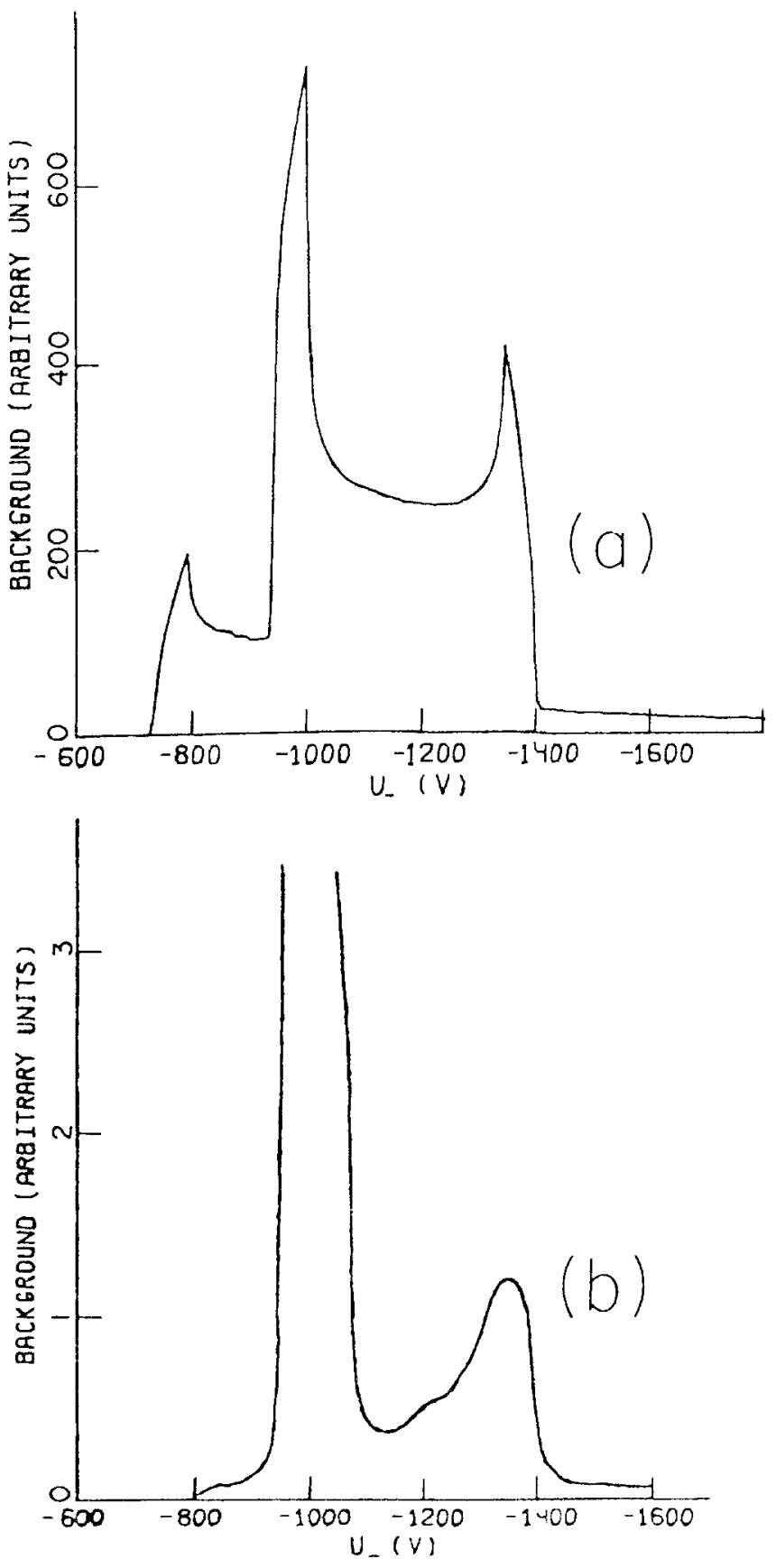

Fig. 9. Dependence of the background on the potentials $U_{-}=$ $-U_{+}$at the cylindrical electrodes for the longitudinal-field ionizer, for $U_{\mathrm{i}}=2000 \mathrm{~V}, U_{\mathrm{f}}=-2000 \mathrm{~V}$ : (a) calculation; (b) experimental data

gitudinal ionization is preferential for the detection of rare isotopes if subsequent mass separation is used.

\section{References}

1. Yu.A. Kudryavtsev, V.S. Letokhov: Appl. Phys. B 29, 219 (1982)

2. S.A. Aseyev, Yu.A. Kudryavtsev, V.S. Letokhov, V.V. Petrunin: Opt. Lett. 16, 514 (1991)

3. S.L. Kaufman: Opt. Commun. 17, 309 (1976)
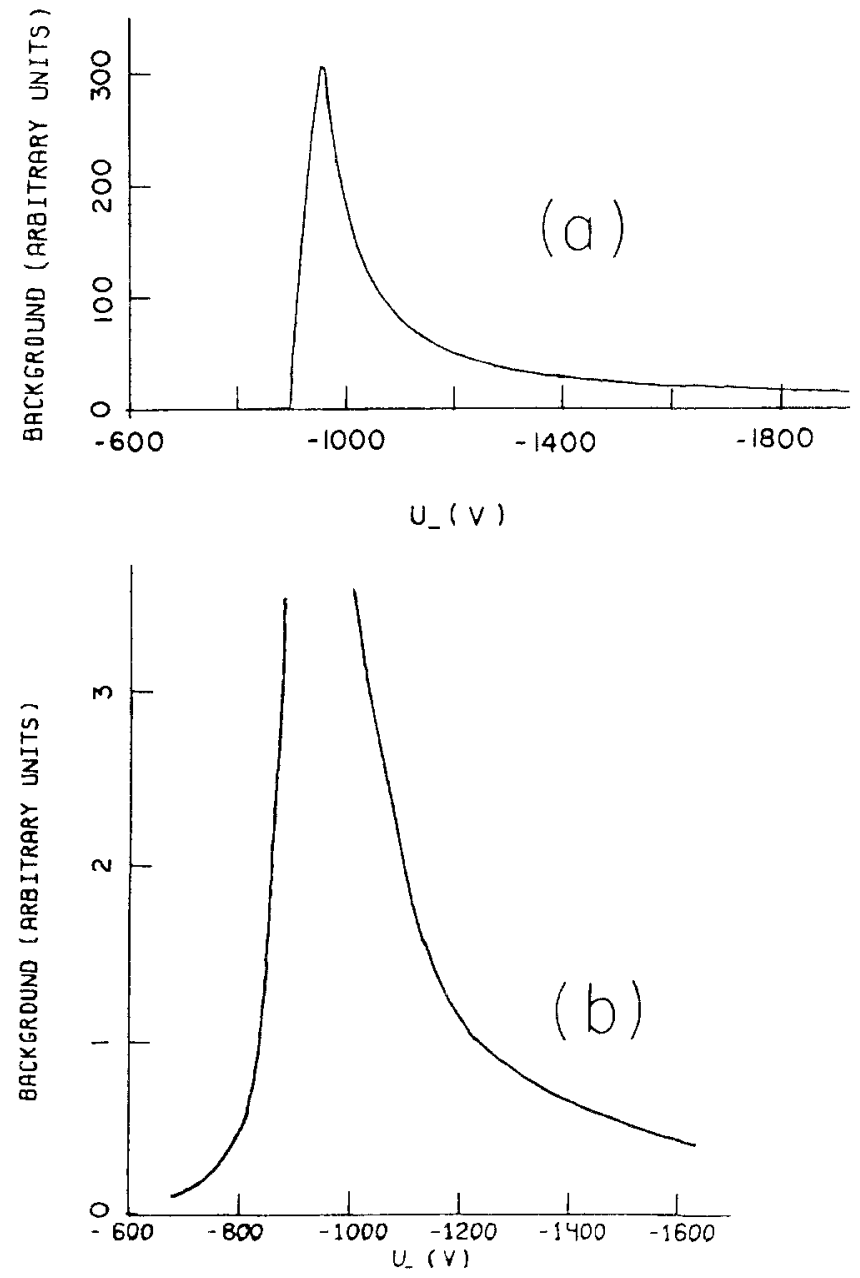

Fig. 10. Dependence of the background on the potentials $U_{-}=$ $-U_{+}$at the cylindrical electrodes for the transverse-field ionizer: (a) calculation; (b) experimental data

4. W.H. Wing, G.A. Ruff, W.E. Lamb, J.J. Spezeski: Phys. Rev. Lett. 36, 1488 (1976)

5. Yu.A. Kudryavtsev, V.V. Petrunin: Zh. Eksp. Teor. Fiz. 94, 76 (1988) (in Russian);

Sov. Phys. JETP 67, 691 (1988)

6. R.N. Il'in, V.A. Oparin, I.T. Serenkov, E.S. Solov'ev, N.V. Fedorenko: Sov. Phys. JETP 32, 59 (1971)

7. A.C. Riviere. In Methods of Experimental Physics, ed. by L. Marton, Vol. 7A (Academic, New-York 1968) p. 208

8. K.B. MacAdom, R.G. Rolfes: Rev. Sci. Instrum. 53, 592 (1982)

9. R.G. Rolfes, K.B. MacAdom: J. Phys. B: At. Mol. Phys. 15, 4591 (1982)

10. U. Dinger, J. Eberz, G. Huber, H. Lochman, G. Ulm, T. Kühl: Z. Phys. D-Atoms, Molecules and Clusters 1, 137 (1986)

11. Ch. Schulz, E. Arnold, W. Borchers, W. Neu, R. Neugart, M. Neuroth, E.W. Otten, M. Scherf, K. Wendt, P. Lievens, Yu.A. Kudryavtsev, V.S. Letokhov, V.I. Mishin, V.V. Petrunin and the ISOLDE collaboration: In Proc. 5th Int'l Symp. on Resonance Ionization Spectroscopy and its Applications (1990) p 27;

J. Phys. B: 24, 4831 (1991)

12. Yu.A. Kudryavtsev, V.S. Letokhov, V.V. Petrunin: Sov. Phys. JETP Lett. 42, 29 (1985) 
13. N. Bendali, H.T. Duong, P. Juncar, J.K.P. Lee, J.M. SaintJalm, J.L. Vialle: J. Physique 47, 1167 (1986)

14. M. Pellarin, J-L Vialle, M. Carre, J. Lerme, M. Aymar: J. Phys. B: At. Mol. Opt. Phys. 21, 3833 (1988)

15. Yu.A. Kudryavtsev, V.V. Petrunin: Zh. Eksp. Teor. Fiz. 99, 81 (1991) (in Russian); Sov. Phys. JETP 72, 43 (1991)

16. S.A. Aseyev, Yu.A. Kudryavtsev, V.V. Petrunin: J. Phys. B: 24, L647 (1991)

17. S.I. Molokovski, A.D. Sushkov: Intensive Electron and Ion Beams (Energiya 1972) (in Russian)
18. V.A. Govorkov: Electric and Magnetic Fields (Energiya, Moscow 1968) (in Russian)

19. D. Kleppner, M.G. Littman, M.L. Zimmerman: In Rydberg States of Atoms and Molecules, ed. by R.F. Stebbings, F.B. Dunning(Cambridge Univ. Press, Cambridge 1983) Chap. 3

20. F.B. Dunning, R.F. Stebbings: In Rydberg States of Atoms and Molecules, ed. by R.F. Stebbings, F.B. Dunning (Cambridge Univ. Press, Cambridge 1983) Chap. 9

Responsible for Advertisements: E. Lückermann, G. Probst, Heidelberger Platz 3, D-1000 Berlin 33, Tel. (0 30) 82 07-0, Telex 01-85411, FAX (0) 30/8207300 Responsible for the Text: H. K. V. Lotsch, Tiergartenstraße 17, D-6900 Heidelberg, Tel. (0) 62 21/487253, Telex 461723, FAX (0) 6221/43982

Printers: Brühlsche Universitätsdruckerei, Giessen

(C) by Springer-Verlag Berlin Heidelberg 1993. Printed in Germany

Springer-Verlag GmbH \& Co. KG, D-1000 Berlin 33 